\title{
Thermal Properties Of Sediments From Middle Valley
}

\author{
A. Hafaiedh, K. Hassine \\ Department of Mechanical Engineering, \\ Institute of High Technology, Nabeul, Tunisia. \\ Tel: 0096823299826 hafaeidhabdel@yahoo.com \\ Department of Computer Sciences, \\ University of Sciences, Monastir, Tunisia. \\ Tel:0096823291528 Khaled_hassine@yahoo.fr
}

\begin{abstract}
:
A neural network model was used to treat thermal conductivity data obtained from Middle Valley. This technique was able to separate the effects of different parameters such as porosity, grain density, bulk density and water content on the thermal conductivity. Predicted curves showed good agreement with the experimental results.
\end{abstract}

Keywords: neural network, thermal conductivity, porosity, grain density, bulk density, water content.

\section{Introduction}

Effective thermal conductivity is an important diffusive transport coefficient to evaluate the coupled heat and moisture transfer through porous walls so that conduction heat fluxes may be precisely calculated. This heat transport coefficient in a porous material may be described in terms of the conductivity of solid matrix and fluid phases and their quantities, phase change phenomena, and special organization of the phases. The present work deals with the use of the neural network techniques in order to predict the dependence of the thermal conductivity as a function of the porosity content for seafloor massive sulfide deposits.

The marked influence of the different characteristics of the seafloor massive sulfide bodies and surrounding seafloor materials on the thermal conductivity measurements opens new directions for field and modeling studies of the combined roles of conduction and convection in heat transfer at seafloor hydrothermal sites.

There have been extensive experimental and analytical work to investigate the characteristics and properties of the massive sulfide mounds deposited at major seafloor hydrothermal sites[1-3] Some of the more interesting work was produced on samples from the Mid-Atlantic Ridge and the Middle Valley in the Pacific. Rona[2] produced experimental work on the thermal conductivity of sulfides/sulfates samples from the volcanic-hosted active sulfide mound in the TAG hydrothermal field in the rift valley of the mid-Atlantic Ridge, using two different methods. Using the half space divided bar method, he found that the thermal conductivity of heterogeneous mixtures of sulfide (predominantly pyrite), quartz, and anhydrite breccias range between 6.1 and $10.4 \mathrm{~W} /(\mathrm{m}$ 
$\left.{ }^{\circ} \mathrm{K}\right)$.

Visual and thin-section observations show that many of the Middle Valley samples used for this study have a matrix rich in clay, silt, and quartz with sulfide veins irregularly distributed throughout the sediments. These veins as well as the massive sulfide samples are composed of approximately equal amounts of pyrite and pyrrhotite. It appears that the matrix composition plays a significant role in the thermal conductivity of hydrothermal deposits. In particular, the presence or lack of anhydrite within the rock matrix seems to affect the measurements the most.

\section{The ANN Model}

Artificial neural networks (ANN) are being used in many different fields, such as predictions[4-6], system designs[7], forecasting and estimation[8]. They are composed of simple computational elements operating both in parallel and in sequence, called neurons or nodes, described by figure 1 . Neurons process information based on weighted inputs using their transfer functions and

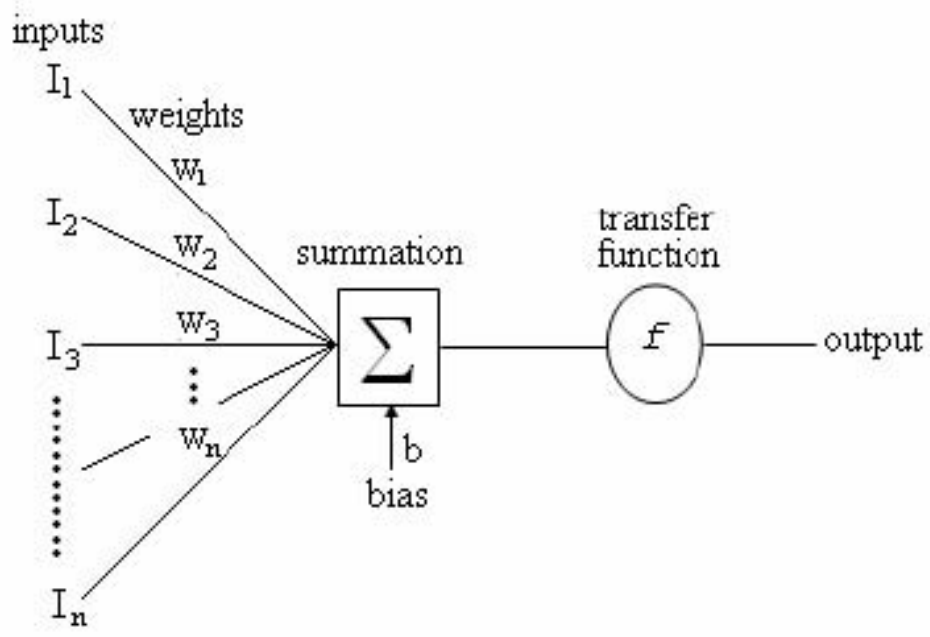

Figure 1: The neuron model sent out outputs. The nodes in adjacent layers, are fully or partially interconnected with weighted links. The weight factors play a critical role during the learning process. They start from an initially random state and move according to the training data sets towards a stable state in a way to minimize the difference between the actual outputs (target) and the model predicted outputs. The transfer function of processing nodes is used to determine the output value of the node based on the total net input from nodes in the prior layer. There are many different neural net types with each having special properties, so each problem domain has its own net type. Based on the topology, the connections of an ANN could be either feedback, where the connections between the nodes form cycles, or feed-forward. The presence of one or more hidden layers is one of the most important characteristics of ANN network. The function of neurons in a hidden layer (hidden neurons) is to intervene between the external input and the network output, in some useful manner, in order to make the output values converge as close as possible to the target values. The program keeps adjusting the values of the weights to allow a minimum value of the mean sum square of the error, then uses the obtained weights in order to calculate the target value. Using more hidden layers enables the network to extract higher order statistics, in 
particular when the number of the input data is large.

In order to build an ANN model, the number of nodes in both, input and output layers, the number of hidden layers and the number of nodes in each hidden layer, need to be defined first. The number of nodes in both input and output layers may be defined based on the problem to be studied. The number of hidden layers and hidden nodes may be determined either by the trial and error approach or using some empirical formulas, as guidance. Kolomogorov's theorem states that twice the number of input variables plus one is enough hidden nodes to compute any arbitrary continuous function. Carpenter introduced an equation to define the number of nodes in the hidden layer based on the number of inputs, and the number of training sample set.

$$
N_{h}=\left(N_{d} / \beta-N_{o}\right) /\left(N_{i}+N_{o}\right)
$$

Where $N_{h}$ is the number of hidden nodes; $N_{d}$ is the number of training data pairs; $\beta$ is the parameter related to the degree over-determination; $N_{i}$ is the number of input nodes; and $N$ is the number of output nodes. In this model, the optimal number of nodes in the hidden layer is determined by trial and error method.

\section{Experimental Procedure}

Measurements were made on vertical and horizontal mini-cores $(2.54 \mathrm{~cm}$ in diameter and $2.54-3.81 \mathrm{~cm}$ in length) from Middle Valley at the Petro-physics laboratory of the Rosenstiel School of Marine and Atmospheric Sciences in Miami, Florida. Wet and dry sample weights and volumes were determined using a microbalance for measuring mass $( \pm 0.03 \%)$.

Porosity values were determined to approximately $\pm 0.2 \%$, with the assumption that the porosity is interconnected and the fluid is saturated. The necessary information to calculate the density porosity and water content was provided by a specifically designed pycnometer, which employs Archimedes principle of fluid displacement. The displaced fluid was helium, which assures penetration into crevices and pore spaces in the order of $1 \AA\left(10^{-10} \mathrm{~m}\right)$. Purge times for $5 \mathrm{~min}$, were made, in order to approach a helium-saturated steady-state condition. After measuring the wet weights, water was driven off, by keeping samples at a temperature of $100^{\circ} \mathrm{C}$ for $24 \mathrm{hr}$.

Measurements were corrected for salt, assuming a pore-water salinity of $35 \%$, as required by the American Society for Testing and Materials (ASTM) (D2216, 1989). Samples were saturated in seawater and placed in a vacuum for $24 \mathrm{hr}$ in order to achieve situ wet conditions.

The ANN configuration, proposed in this work, has the values of the depth, the bulk density, the grain density, the pore water volume, and the porosity as inputs and the corresponding thermal conductivities as outputs. Using this input-output arrangement, we tried many different network configurations to achieve good performance of the network. 
The, five layer, back-propagation model, was considered. It corresponded to the lowest value of the mean sum square of the error. The first layer is the input layer, with 5 neurons, does not have any computing activity. It was, simply used to enter the values of the input parameters. The second, third and fourth layers are hidden layers with seven, five and five neurons, respectively. The fifth layer is the output layer with one neuron, used to process the outcome for the thermal conductivity. We have chosen the Levenberg-Marquardt optimization technique[9], which is a powerful algorithm, as a training algorithm, the log sigmoid function as a transfer function for the first and second layers and the linear sigmoid function as a transfer function for the output layer.

\section{Results and discussion}

Table 1 describes the experimental results obtained for 41 Middle Valley samples. The pore water volume, PWV, is the ratio of the mass of water in a sediment sample to the mass of the wet sample. As described by figure 2, PWV shows an almost proportional dependence on the percent porosity. Equation 1 describes the relationship, used to calculate the pore water volume for any percent porosity during modeling, which was determined using a linear fitting program.

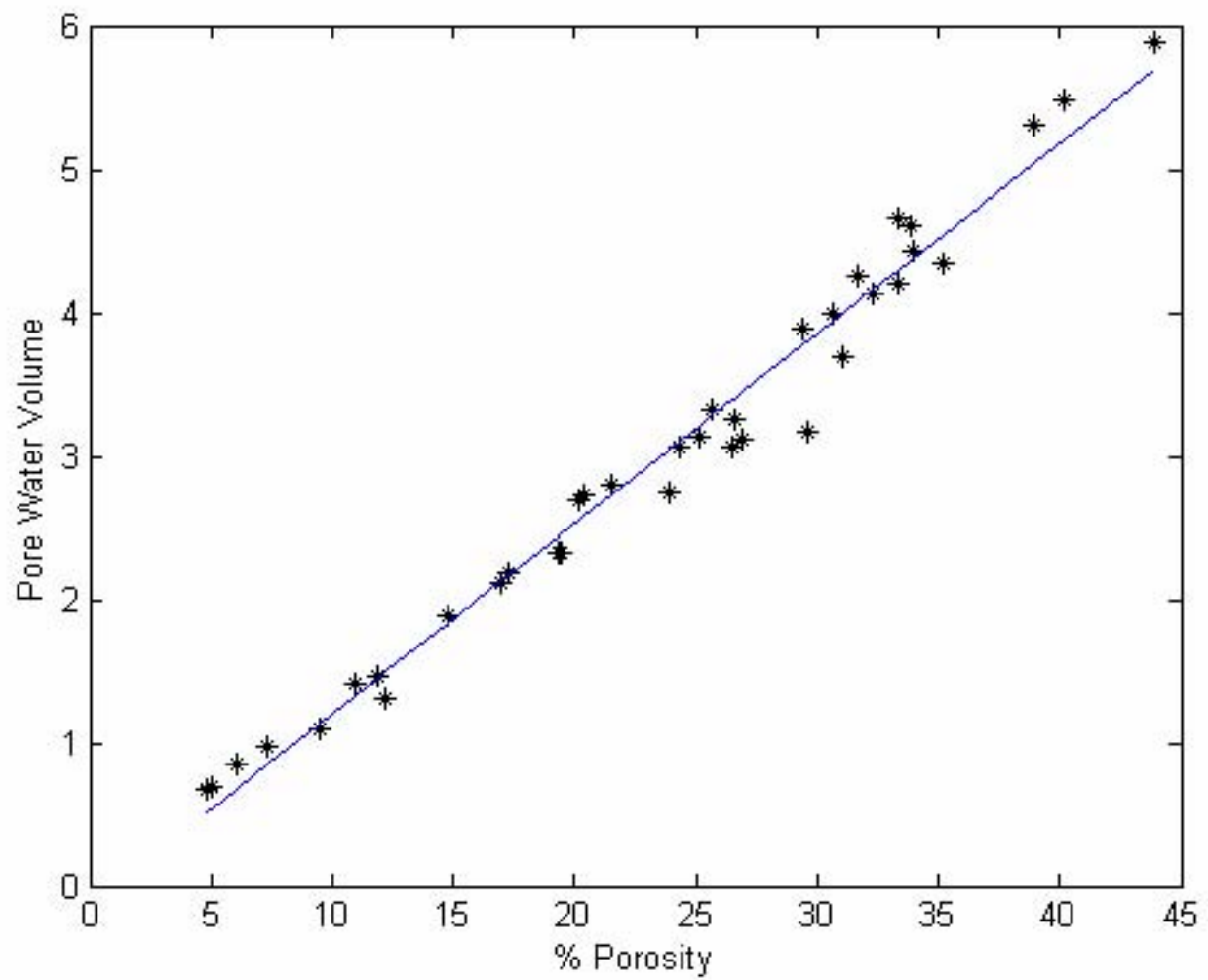

Figure 2: Dependence of the pore water volume on the percent porosity. The solid line was determined by linear fitting 
Table 1: Index properties and thermal conductivities of samples from the Middle Valley[1].

\begin{tabular}{|c|c|c|c|c|c|}
\hline$\#$ & $\begin{array}{c}\text { Bulk Density } \\
(\mathrm{g} / \mathrm{cm} 3)\end{array}$ & $\begin{array}{c}\text { Grain Density } \\
(\mathrm{g} / \mathrm{cm} 3)\end{array}$ & $\begin{array}{c}\text { Pore Water } \\
\text { Volume }(\mathrm{cm} 3)\end{array}$ & \% Porosity & $\begin{array}{c}\text { Thermal Conductivity } \\
\left(\mathrm{W} /\left(\mathrm{m}^{\circ} \mathrm{K}\right)\right)\end{array}$ \\
\hline 1 & 2.86 & 2.96 & 0.67 & 4.82 & 2.48 \\
\hline 2 & 2.86 & 2.96 & 0.70 & 4.98 & 2.42 \\
\hline 3 & 2.75 & 2.86 & 0.85 & 6.07 & 3.91 \\
\hline 4 & 3.85 & 4.07 & 0.97 & 7.31 & 4.11 \\
\hline 5 & 2.69 & 2.85 & 2.02 & 8.41 & 4.92 \\
\hline 6 & 2.63 & 2.80 & 1.09 & 9.49 & 5.10 \\
\hline 7 & 2.66 & 2.87 & 1.42 & 10.91 & 4.67 \\
\hline 8 & 2.77 & 3.02 & 1.31 & 12.18 & 3.98 \\
\hline 9 & 4.32 & 4.81 & 1.61 & 12.75 & 10.95 \\
\hline 10 & 4.19 & 4.75 & 1.89 & 14.78 & 7.18 \\
\hline 11 & 2.48 & 2.83 & 2.33 & 19.30 & 3.36 \\
\hline 12 & 2.44 & 2.79 & 2.33 & 19.49 & 3.99 \\
\hline 13 & 4.00 & 4.75 & 3.60 & 19.92 & 7.75 \\
\hline 14 & 2.58 & 2.99 & 2.72 & 20.40 & 3.05 \\
\hline 15 & 2.37 & 2.80 & 2.74 & 23.90 & 2.99 \\
\hline 16 & 2.42 & 2.88 & 3.06 & 24.32 & 3.57 \\
\hline 17 & 3.52 & 4.36 & 3.14 & 25.11 & 3.42 \\
\hline 18 & 2.32 & 2.81 & 3.07 & 26.49 & 3.28 \\
\hline 19 & 2.31 & 2.79 & 3.26 & 26.56 & 3.17 \\
\hline 20 & 2.00 & 2.74 & 3.11 & 26.9 & 3.03 \\
\hline 21 & 2.30 & 2.85 & 3.88 & 29.40 & 3.23 \\
\hline 22 & 2.27 & 2.81 & 3.17 & 29.60 & 3.00 \\
\hline 23 & 1.86 & 2.69 & 3.69 & 31.10 & 2.95 \\
\hline 24 & 2.27 & 2.89 & 4.13 & 32.35 & 2.89 \\
\hline 25 & 2.21 & 2.81 & 4.20 & 33.29 & 2.85 \\
\hline 26 & 2.20 & 2.80 & 4.66 & 33.36 & 3.45 \\
\hline 27 & 2.21 & 2.84 & 4.43 & 33.92 & 3.57 \\
\hline 28 & 2.10 & 2.81 & 5.31 & 38.93 & 2.84 \\
\hline 29 & 2.11 & 2.87 & 5.49 & 40.20 & 2.53 \\
\hline 30 & 2.06 & 2.89 & 5.89 & 43.87 & 2.64 \\
\hline & & & & & \\
\hline
\end{tabular}

$$
P W V=0.1272 P+0.0509
$$

Grain density is the ratio of the bulk density minus the porosity to the complement of the porosity as shown by equation 2 . Equation 3 describes the dependence of the bulk density on both porosity and grain density.

$$
\begin{aligned}
& G D=\frac{B D-P}{1-P} \\
& B D=G D(1-P)+P
\end{aligned}
$$

Where GD is the grain density, $\mathrm{BD}$ is the bulk density, and $\mathrm{P}$ is the percent porosity. The dependences of the thermal conductivity as a function of percent porosity and grain 
density are described in figures $3 a$ and $3 b$, respectively. Results show no clear correlations, which may be due to the fact that the controlling parameters of the thermal dissipation of heat are changing simultaneously, making a separate investigation of their effects impossible.

The ANN model was trained using data in table 1 . The depth, the bulk density, the grain density, the pore water volume, and the porosity were the dependent parameters whereas the thermal conductivity was the target values. Results of the ANN model were then, used in order to predict the dependence of the thermal conductivity on its influencing parameters. In all predictions, the values of the bulk density were determined using equation 3 .

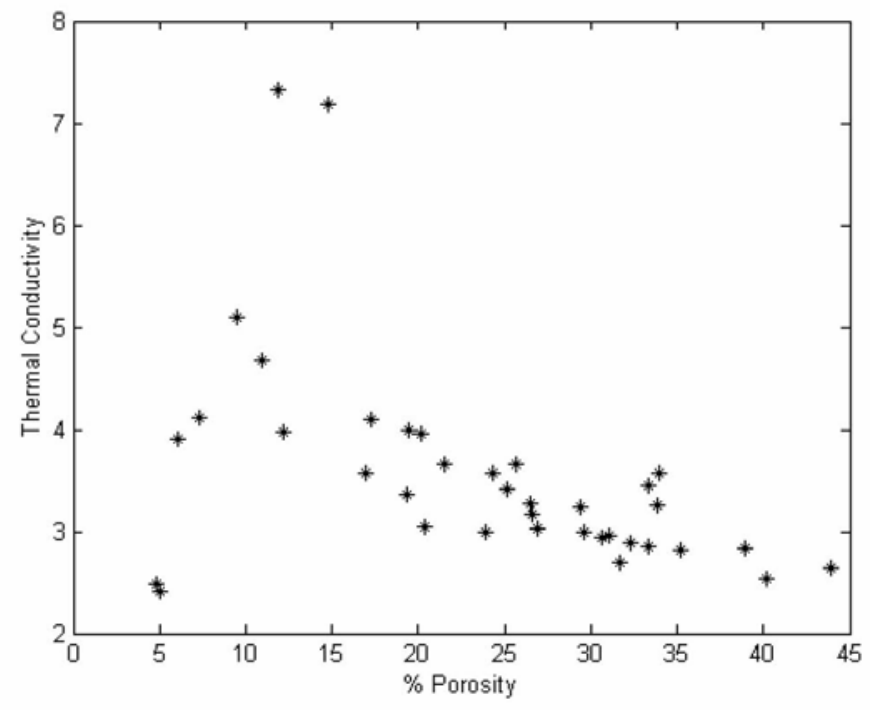

Figure 3a: Dependence of the thermal conductivity on the porosity (experimental results)

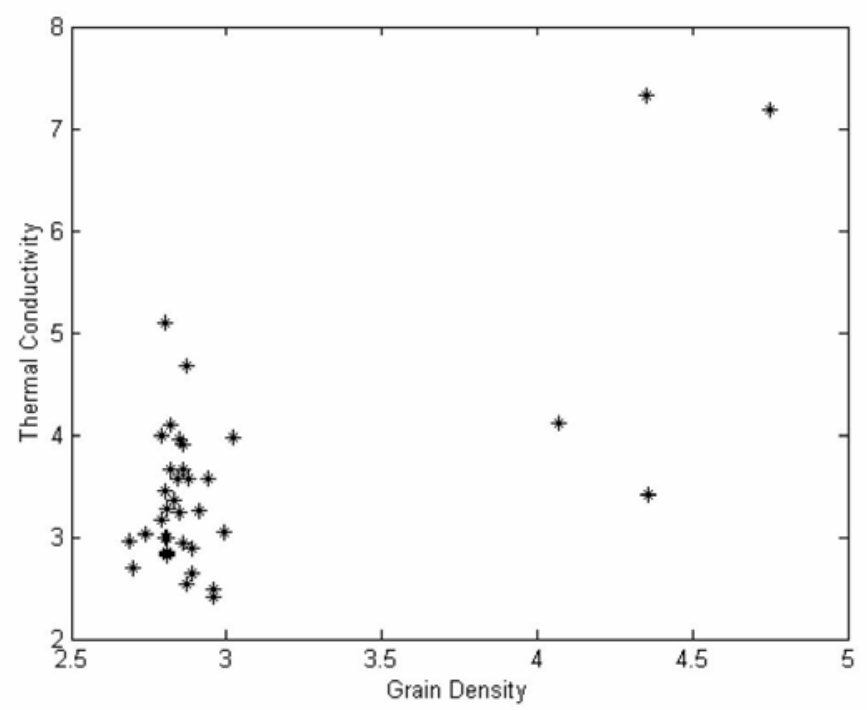

Figure 3b: Dependence of the thermal conductivity on the grain density fordifferent values of the porosity (experimental results)

\section{Effects of the percent porosity}

Figure $4 \mathrm{a}$ describes the dependence of the thermal conductivity on the porosity, for different values of the grain density. In this case, the values of the pore water volume were determined from the porosity (equation 1). We notice, first a rapid decrease of the thermal conductivity as a function of the porosity, then decreases slowly. Figure $4 \mathrm{~b}$ describes the dependence of the thermal conductivity on the percent porosity for constant values of the pore water volume. The thermal conductivity decreases rapidly up to a 
somewhat constant value. Higher grain density values allowed higher values of the thermal conductivity.

\section{Effects of grain density}

Figure 5 describes the effects of the grain density on the thermal conductivity for different values of the porosity. The thermal conductivity keeps an almost constant value then increases rapidly. This behavior may be because grains may have some internal porosity, which increases as the grain density decreases.

\section{Conclusions}

In this wok, we used the neural technique in order determine the dependence of the thermal conductivity of Middle Valley samples on some different parameters such as porosity, grain density and water content. This technique proved to be able to separate the effects of the different parameters on the thermal conductivity, which is impossible to realize experimentally. Effects of other parameters such as phase composition, grain size distribution and pore size distribution could be determined and used in the training procedures for a better prediction capability.

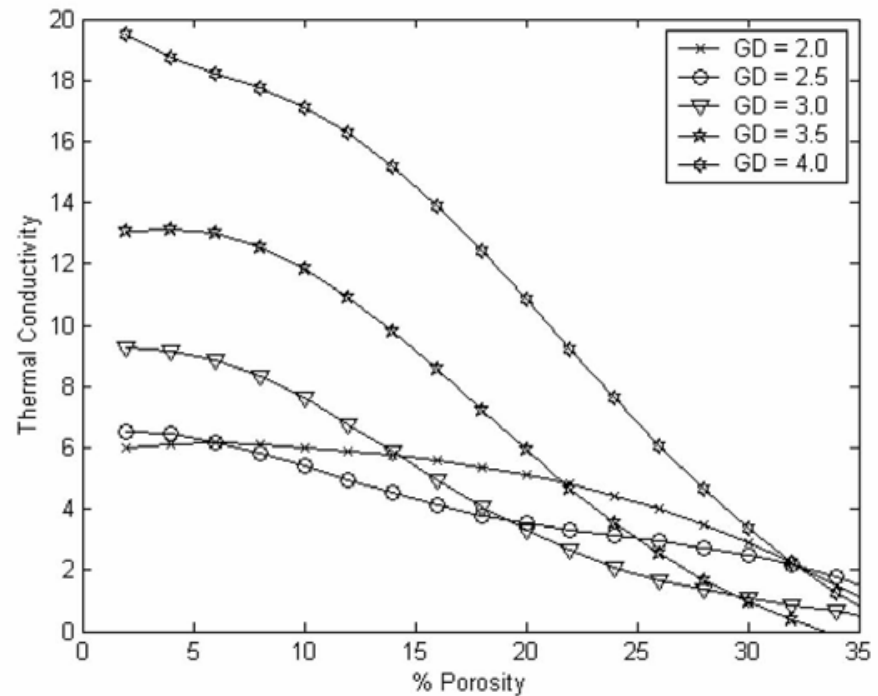

Figure 4a: Dependence of the thermal conductivity on the porosity for different values of the grain density. In this case, the value of the pore water volume is determined using equation 1

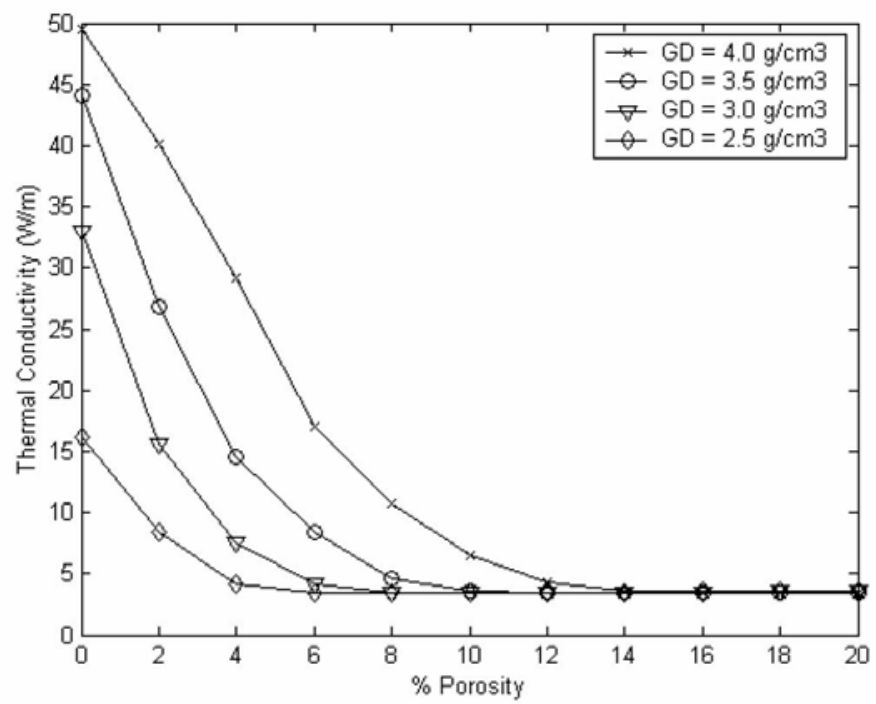

Figure 4b: Dependence of the thermal conductivity on the Porosity for different values of the grain density. In this case, the value of the pore water volume is constant 


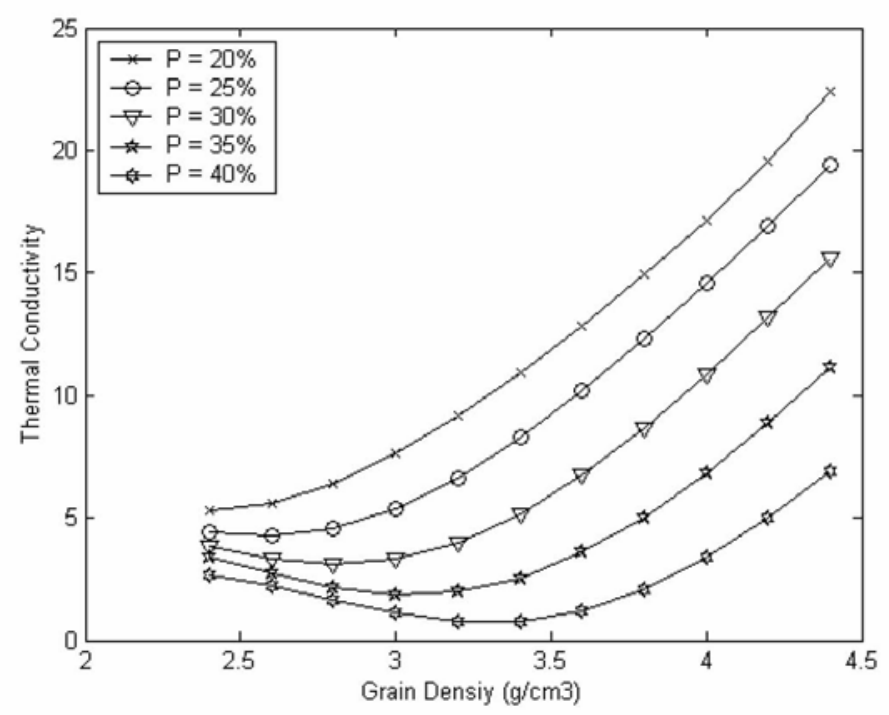

Figure 5: Dependence of the thermal conductivity on the grain density for different values of the porosity

\section{References}

[1] Rona. P.A., Davis, E.E., and Ludwig, R.J., "Thermal properties of TAG hydrothermal precipitates, Mid-Atlantic Ridge: a comparison with Middle Valley, Juan de Fuca Ridge". In Herzig, P.M., Humphris, S.E., Miller, D. J., and Zierenberg, R.A. editors, 1998, Proc. ODP, Sci. Results, 158: College Station, TX (Ocean Drilling Program), 329-335.

[2] Davis, E.E., and Villinger, H., "Tectonic and thermal structure of the Middle Valley sedimented rift, northern Juan de Fuca Ridge". In Davis, E.E., Mottl, M.J., Fisher, A.T., et al., editors, 1992, Proc. ODP, Init. Repts., 139, College Station, TX (Ocean Drilling Program), 9-41.

[3] Gröschel-Becker, H.M., Davis, E.E., and Franklin, J.M., 1994. Data Report: Physical properties of massive sulfide from Site 856, Middle Valley, northern Juan de Fuca Ridge. In Mottl, M.J., Davis, E.E., Fisher, A.T., and Slack, J.F. (Eds.), Proc. ODP, Sci. Results, 139: College Station, TX (Ocean Drilling Program), 721-724.

[4] M. Y. El-Bakry, K. A. El-Metwally, 2003, "Neural network model for proton-proton collision at high energy." Chaos, Solitons and Fractals, No. 16, pp. 279-285.

[5] M. Lee, S. Hwang, and J. Chen, 1994, "Density and Viscosity Calculations for Polar Solutions via Neural Networks." J. Chem. Eng. of Japan, Vol. 27, No. 6, pp. 749754.

[6] Y. Baram and Z. Roth, 1994 "Density shaping by neural networks with application to classification, estimation and forecasting" Center for Intelligent Systems," Israel Institute for Technology, Haifa, Israel, Tech. Rep. CIS-94-20.

[7] Y. Sun, Y. Pengand, A. Shukla, 2003, "Application of Artificial Neural Networks in the Design of Controlled Release Drug Delivery Systems." Advanced Drug Delivery Reviews No. 55, pp. 1201-1215. 
[8] J. Bourquin, H. Schmidli, P. Van Hoogevest, and H. Leuenberger, 1997, "Application of Artificial Neural Networks (ANN) in the Development of Solid Dosage Forms." Pharm. Dev. Technol., No. 2, pp.111-121

[9] M.T. Hagan and M.B. Menhaj, 1994, Training feed-forward networks with the Marquardt algorithm." IEEE Transactions on Neural Networks, No. 6, pp. 861-867. 